\title{
A Population Pharmacokinetic and Exposure-Response Model of Golimumab for Targeting Endoscopic Remission in Patients With Ulcerative Colitis
}

\author{
Erwin Dreesen, MSc, PharmD, * Wannee Kantasiripitak, PharmD, * Iris Detrez, PharmD, PhD,* \\ Sebastian Stefanović, MD, ${ }^{+}$Séverine Vermeire, MD, PhD, ${ }^{\neq}$Marc Ferrante, MD, PhD, ${ }^{\ddagger}$ Thomas Bouillon, MD, \\ $P h D,{ }^{*}$ David Drobne, MD, PhD, ${ }^{+}$and Ann Gils, PharmD, PhD*
}

Background: Unlike other anti-tumor necrosis factor alpha antibodies, golimumab does not deliver on its promise of effectiveness for treating patients with ulcerative colitis. We investigated the value of therapeutic drug monitoring for optimizing golimumab therapy.

Methods: We analyzed the golimumab pharmacokinetics data of 56 patients with moderate to severe ulcerative colitis. Induction and maintenance golimumab concentrations (296 venipuncture, 414 serum) were used to develop a population pharmacokinetic model. Exposure-response relationships were analyzed using the data of $40 / 56$ patients with available endoscopy data. Receiver operating characteristic curve analysis was performed, and an exposure-response Markov model was developed, linking golimumab exposure to probabilities of transitioning between Mayo endoscopic subscore (MES) states from baseline to week (w)14.

Results: Golimumab pharmacokinetics was best described by a 2-compartment model with linear absorption and elimination. Antibodies to golimumab and previous biological therapy reduced golimumab exposure. Still, interindividual pharmacokinetic variability (IIV $\mathrm{PK}_{\mathrm{PK}}$ ) remained largely unexplained. Endoscopic remission (ER; $\left.\mathrm{MES}_{\mathrm{w} 14} \leq 1\right)$ was achieved in 14/40 (35\%) patients. Golimumab serum trough concentration thresholds of $7.4 \mathrm{mg} / \mathrm{L}$ (w6) and $3.2 \mathrm{mg} / \mathrm{L}$ (w14) predicted ER at w14 (positive predictive values [pv+] 83\% and 91\%, pv- 82\% and 67\%, respectively). The $3.2-\mathrm{mg} / \mathrm{L}$ target predicted $38 \%$ and $44 \%$ chances of achieving ER in patients with $\mathrm{MES}_{\text {baseline }}$ of 3 and 2 , respectively.

Conclusions: Personalized, model-based induction dosing aiming at here-established target concentrations may account for IIV ${ }_{\text {PK }}$ and thus provide patients with more equal chances of achieving ER. As $<50 \%$ of patients attained the exposure targets, higher golimumab induction dosing requires investigation to secure its future in clinical practice.

Key Words: monoclonal antibodies, therapeutic drug monitoring, personalized medicine, exposure response, population pharmacokinetics-pharmacodynamics

\section{INTRODUCTION}

Golimumab is a fully human immunoglobulin G1 monoclonal antibody that inhibits tumor necrosis factor alpha $(\mathrm{TNF} \alpha){ }^{1}$ Based on the integrated phase 2b/3 Program of Ulcerative Colitis Research Studies Utilizing an Investigational Treatment (PURSUIT) studies, golimumab was approved for

Received for publications March 27, 2019

From the *Department of Pharmaceutical and Pharmacological Sciences, University of Leuven, Leuven, Belgium; 'Department of Gastroenterology and Hepatology, University Medical Centre Ljubljana, Ljubljana, Slovenia; ${ }^{\star}$ Department of Gastroenterology and Hepatology, University Hospitals Leuven, Leuven, Belgium

Supported by: This work was supported by the Research Foundation - Flanders (Fonds Wetenschappelijk Onderzoek [FWO] - Vlaanderen), Belgium (TBM grant T003716N)

Conflicts of interest: S.V. and M.F. are senior clinical researchers of the Research Foundation Flanders (FWO). S.V. received grant support from Merck Sharp \& Dohme, AbbVie, Pfizer, and Takeda, lecture fees from AbbVie, Merck Sharp \& Dohme, Ferring Pharmaceuticals, Takeda, and Hospira, and consultancy fees from AbbVie, Takeda, Pfizer, Ferring Pharmaceuticals, Shire Pharmaceuticals Group, Merck Sharp \& Dohme, Hospira, Mundipharma, Celgene, Galapagos, and Genentech/Roche. M.F. received financial support for research from Takeda, lecture fees from Merck Sharp \& Dohme, Janssen Pharmaceutica, AbbVie, Boehringer Ingelheim, Ferring Pharmaceuticals, Chiesi, Tillotts, Zeria, and Mitsubishi Tanabe, and consultancy fees from Merck Sharp \& Dohme, Janssen Pharmaceutica, AbbVie, Boehringer Ingelheim, and Ferring Pharmaceuticals. D.D. received lecture and the treatment of adults with moderate to severe ulcerative colitis (UC) who have not responded adequately to or cannot use conventional therapies. ${ }^{2-4}$ The PURSUIT-SC study showed that subcutaneous golimumab administration safely induces clinical response, clinical remission, and endoscopic remission and increases quality of life at week 6 in larger percentages of

consultancy fees from Merck Sharp \& Dohme, AbbVie, Takeda, Pfizer, Janssen Pharmaceutica, Krka, Dr. Falk Pharma, and Ferring Pharmaceuticals. A.G. received speaker fees from Merck Sharp \& Dohme, Janssen Biologicals, Pfizer, Takeda, and AbbVie, consultancy fees from UCB, investigator-initiated research grants from Pfizer, and a national grant from Takeda. E.D., W.K., I.D., S.S., and T.B. declare that they have no conflicts of interest

Author contributions: E.D., A.G.: designed the research. E.D., I.D., S.S., S.V., M.F., D.D.: generated and assembled the data. E.D., W.K.: performed the research and analyzed the data. E.D.: wrote the manuscript. W.K., I.D., S.S., D.D., S.V., M.F., T.B., and A.G.: critical revision of the manuscript. All authors approved the final version of the manuscript.

\section{Trial registration: ORCID 0000-0002-0785-2930.}

Address correspondence to: Erwin Dreesen, MSc, PharmD, Therapeutic and Diagnostic Antibodies Unit, O\&N II Herestraat 49 Box 820, 3000 Leuven, Belgium (erwin.dreesen@kuleuven.be).

(C) 2019 Crohn's \& Colitis Foundation. Published by Oxford University Press. All rights reserved. For permissions, please e-mail: journals.permissions@oup.com. doi: 10.1093/ibd/izz144 Published online 2 August 2019 
patients than placebo. ${ }^{3}$ The PURSUIT-M study showed that golimumab was able to maintain the initially induced clinical response and achieve clinical remission and endoscopic remission at both weeks 30 and 54 of therapy. ${ }^{4}$

In PURSUIT, golimumab serum concentrations as early as week 2 were higher among patients achieving clinical response and endoscopic remission at week $6 .^{5}$ Adedokun et al. identified optimal golimumab serum concentration thresholds at weeks 2, 4, and 6 of $8.9 \mathrm{mg} / \mathrm{L}, 7.4 \mathrm{mg} / \mathrm{L}$, and $2.5 \mathrm{mg} / \mathrm{L}$, respectively, as desirable targets for attainment of an optimal clinical outcome rate in the population. In a small observational "realworld" study, Detrez et al. reported higher golimumab serum concentrations at week 6 among patients achieving partial clinical response. ${ }^{6}$ An optimal week 6 golimumab serum concentration threshold of $2.6 \mathrm{mg} / \mathrm{L}$ was identified. Therapeutic drug monitoring (TDM) may be used for targeting these thresholds.

Although symptom resolution and control were the primary therapeutic goals in PURSUIT, treatment goals have evolved in recent years, now largely focusing on endoscopic healing. ${ }^{7}$ Indirect evidence supports the idea that early endoscopic healing is associated with improved long-term clinical outcomes, corticosteroid discontinuation, and lower risk of colectomy. ${ }^{7,8}$ To date, no optimal golimumab serum concentration thresholds have been established for targeting endoscopic remission.

Patients in the PURSUIT studies were biologically naïve, which undermines the relevance of these studies for real-world clinical practice, where patients have often been pre-exposed to infliximab and/or adalimumab. Evidence is growing that the efficacy of biological therapies is inferior in patients who were previously treated with biologicals as compared with those who are biologically naïve. ${ }^{9}$ For this reason, confirmatory data on the effectiveness of golimumab in the real-world setting are needed.

In the PURSUIT-SC and PURSUIT-M studies, golimumab serum concentrations were measured in blood samples collected predominantly at trough. This relatively sparse sampling scheme may not be sufficient to adequately describe the golimumab population pharmacokinetics. ${ }^{10}$ To allow more flexibility in sample collection for building an adequate population pharmacokinetic model, a dried blood spot (DBS) sampling technique has been developed and analytically validated for the quantification of golimumab. ${ }^{11}$

In the current work, we evaluated the role of TDM of golimumab for targeting endoscopic healing in real-world clinical practice. Therefore, we developed a population pharmacokinetic model and an exposure-response model to describe the golimumab dose-exposure and exposure-response relationship, respectively, and to potentially facilitate model-based dose optimization for targeting the established exposure and response targets.

\section{METHODS}

\section{Study Population}

Adult patients with a confirmed diagnosis of UC at 2 tertiary hospitals receiving golimumab between November 2015 and September 2017 were included. The study designs and patient inclusion criteria for these studies have been previously described. ${ }^{6,11,12}$ Study 1 was a prospective study performed at the University Hospitals Leuven, Belgium, including 10 adult patients with UC on golimumab therapy, 5 starting induction therapy and 5 on maintenance therapy. ${ }^{11}$ Study 2 was a retrospective study, also performed at the University Hospitals Leuven, Belgium, including 21 patients starting golimumab therapy. ${ }^{6}$ Study 3 was a retrospective study performed at the University Medical Centre Ljubljana, Slovenia, including 24 patients starting golimumab therapy. ${ }^{12}$ After publication of the original studies, an additional 3 and 3 patients were included in studies 2 and 3 . As 5 patients were included in both study 1 and study 2, our pooled data analysis included a total of 56 patients.

\section{Dosing Scheme}

Golimumab was administered subcutaneously, after a 2-dose induction regimen with fixed doses of $200 \mathrm{mg}$ at baseline (day 0) and $100 \mathrm{mg} 14$ days later. Maintenance therapy followed body weight-based stratified dosing at 4-week intervals from week 6 (day 42) onwards, involving 50-mg doses for patients weighing $<80 \mathrm{~kg}$ and $100-\mathrm{mg}$ doses for patients weighing $\geq 80 \mathrm{~kg}$. Patients who had an inadequate response upon induction therapy received 100-mg maintenance doses, irrespective of body weight.

\section{Sampling Scheme}

In study 1, capillary DBS samples were collected after a rich sampling scheme (Table 1; Supplementary Fig. 1) ${ }^{11}$ In study 2 , peripheral venous serum samples were taken at days 7 , $14,28,42$, and $98 .{ }^{6}$ In study 3 , peripheral venous serum samples were taken at days 3, 7, 14, 21, 28, 42, 70, 98, 182, 266, 280, 308 , and $350 .{ }^{12}$ DBS eluates and serum samples were stored at $-20^{\circ} \mathrm{C}$, under which condition immunoglobulin $\mathrm{G}$ has been shown to be stable for up to 25 years. ${ }^{13}$

\section{Analytical Methods}

Golimumab concentrations in serum and DBS were determined by a validated enzyme-linked immunosorbent assay (ELISA). ${ }^{6,11}$ The lowest quantifiable concentration was $0.1 \mathrm{mg} / \mathrm{L}$. Antibodies to golimumab (ATG) were quantified in serum samples using a validated drug-tolerant ELISA. ${ }^{6}$ The lowest quantifiable concentration was $25 \mathrm{ng} / \mathrm{mL}$ equivalents of monoclonal antibody to golimumab 159B8. Given the relative drug tolerance of the ATG assay, ATG were only quantified in samples with a golimumab concentration of $\leq 4.0 \mathrm{mg} / \mathrm{L}$.

\section{Efficacy Evaluation}

Endoscopic remission was defined as going from a baseline Mayo endoscopic subscore of 2 (moderate disease) or 3 (severe disease) to a subscore of 0 (inactive disease) or 1 (mild disease) at week 14 (day 98). 
TABLE 1. Data Summary

\begin{tabular}{|c|c|c|c|c|}
\hline Parameter & Study 1 & Study 2 & Study 3 & Pooled \\
\hline No. patients $(\%)$ & $10(18)$ & $19(34)$ & $27(48)$ & $56(100)$ \\
\hline \multicolumn{5}{|l|}{ Baseline demographics } \\
\hline Sex, female, No. $(\%)$ & $6(60)$ & $9(47)$ & $15(56)$ & $30(54)$ \\
\hline Age, median (IQR), y & $35(24-54)$ & $45(31-53)$ & $45(39-55)$ & $45(33-54)$ \\
\hline Weight, median (IQR), kg & $74(60-80)$ & $71(65-76)$ & $75(65-87)$ & $72(64-82)$ \\
\hline Fat-free mass, median (IQR), $\mathrm{kg}$ & $45(39-59)$ & $47(42-57)$ & $50(43-60)$ & $47(41-58)$ \\
\hline Previous biological therapy, No. (\%) & $1(10)$ & $8(42)$ & $2(7)$ & $11(20)$ \\
\hline Infliximab, No. (\%) & $0(0)$ & $5(26)$ & $0(0)$ & $5(9)$ \\
\hline Adalimumab, No. (\%) & $1(10)$ & $3(16)$ & $2(7)$ & $6(11)$ \\
\hline \multicolumn{5}{|l|}{ Reason stop previous biological therapy } \\
\hline Primary nonresponse, No. (\%) & $1(10)$ & $1(5)$ & $0(0)$ & $2(4)$ \\
\hline Loss of response, No. $(\%)$ & $0(0)$ & $5(26)$ & $1(4)$ & $6(11)$ \\
\hline Intolerance, No. $(\%)$ & $0(0)$ & $2(11)$ & $1(4)$ & $3(5)$ \\
\hline Current smokers, No. $(\%)$ & $2(20)$ & $0(0)$ & $2(7)$ & $4(7)$ \\
\hline \multicolumn{5}{|l|}{ Serology at baseline } \\
\hline C-reactive protein, median (IQR), mg/L & $3.8(0.5-5.4)$ & $4.8(1.2-22.1)$ & $5.0(5.0-6.5)$ & $5.0(4.6-8.8)$ \\
\hline Albumin, median (IQR), g/L & $43.0(41.8-44.9)$ & $42.1(39.8-44.8)$ & $43.0(41.5-45.0)$ & $43.0(40.9-45.0)$ \\
\hline Alkaline phosphatase, median (IQR), U/L & $66.5(60.2-80.8)$ & $69.0(57.0-90.5)$ & $69.0(58.5-72.0)$ & $69.0(58.0-81.5)$ \\
\hline \multicolumn{5}{|l|}{ Concomitant medication } \\
\hline Mesalazine, No. (\%) & $7(70)$ & $9(47)$ & $0(0)$ & $16(29)$ \\
\hline Azathioprine, No. (\%) & $0(0)$ & $2(11)$ & $8(30)$ & $10(18)$ \\
\hline Corticosteroids, No. $(\%)$ & $3(30)$ & $9(47)$ & $10(37)$ & $22(39)$ \\
\hline \multicolumn{5}{|l|}{ Sampling } \\
\hline Samples available, No. (\%) & $376(53)$ & $148(21)$ & $186(26)$ & $710(100)$ \\
\hline Venipuncture, No. (\%) & $80(21)$ & $148(100)$ & $186(100)$ & $414(58)$ \\
\hline Dried blood spot, No. ( $\%)$ & $296(79)$ & $0(0)$ & $0(0)$ & $296(42)$ \\
\hline No. samples per patient, median (range) & $40(31-64)$ & $4(2-13)$ & $8(3-13)$ & $7(2-64)$ \\
\hline $\begin{array}{l}\text { Samples with undetectable golimumab, } \\
\text { No. }(\%)\end{array}$ & $0(0)$ & $0(0)$ & $0(0)$ & $0(0)$ \\
\hline $\begin{array}{l}\text { Samples with antibodies to golimumab, } \\
\text { No. }(\%)\end{array}$ & $12(3.5)$ & $1(1)$ & $2(1)$ & $15(2)$ \\
\hline $\begin{array}{l}\text { Patients with antibodies to golimumab, } \\
\text { No. }(\%)\end{array}$ & $5(50)$ & $1(5)$ & $1(4)$ & $7(13)$ \\
\hline \multicolumn{5}{|l|}{ Endoscopy } \\
\hline $\begin{array}{l}\text { Baseline Mayo endoscopic subscore, } \\
0: 1: 2: 3: \text { NA, No. }(\%)\end{array}$ & $0: 2: 5: 3: 0(0: 20: 50: 30: 0)$ & $0: 0: 7: 12: 0(0: 0: 37: 63: 0)$ & $0: 3: 7: 10: 7(0: 11: 26: 37: 26)$ & $0: 5: 19: 25: 7(0: 9: 34: 45: 13)$ \\
\hline $\begin{array}{l}\text { Postinduction Mayo endoscopic subscore, } \\
0: 1: 2: 3: \text { NA, No. }(\%)\end{array}$ & $\begin{array}{l}3: 1: 2: 3: 1 \\
\quad(30: 10: 20: 30: 10)\end{array}$ & $1: 2: 6: 8: 2(5: 11: 32: 42: 11)$ & $10: 3: 6: 5: 3(37: 11: 22: 19: 11)$ & $\begin{array}{l}14: 6: 14: 16: 6 \\
\quad(25: 11: 25: 29: 11)\end{array}$ \\
\hline $\begin{array}{l}\text { Endoscopic remission, yes:no:NA, No. } \\
(\%)\end{array}$ & $3: 5: 2(30: 50: 20)$ & $3: 14: 2(16: 74: 11)$ & $8: 7: 12(30: 26: 44)$ & $14: 26: 16(25: 46: 29)$ \\
\hline
\end{tabular}

\section{Population Pharmacokinetic and Exposure- Response Model Development and Evaluation}

Population modeling was performed in NONMEM (version 7.4; Icon Development Solutions, Ellicott City, MD, USA) with a GNU Fortran 95 compiler and the Perl-SpeaksNONMEM (PsN; version 4.7.0) toolkit and Pirana (version 2.9.7).

\section{Population pharmacokinetic model}

Different structural models were fitted to the golimumab pharmacokinetic data employing first-order conditional estimation with interaction and subroutine ADVAN6. Models were fitted simultaneously to serum and DBS concentrations by estimation of a population conversion factor that related individually predicted serum and DBS concentrations. ${ }^{14}$ Based on visual 
inspection of the data and a literature review, a pharmacokinetic model was developed starting from a 1-compartment model with linear absorption and elimination. ${ }^{10,15-19}$ The most parsimonious model was withheld based on model fit evaluations using standard goodness-of-fit diagnostics (residual and simulationbased plots), convergence of the minimization criteria, physiological plausibility, precision of the parameter estimates, and a drop in the objective function value (OFV) of at least 3.84 ( $P \leq 0.050$, 1 degree of freedom). All structural model parameters were assumed to be log-normally distributed. Two levels of random effects were estimated: interindividual variability and residual variability. Additive and/or proportional residual error models were explored separately for serum and DBS concentrations. Eta and epsilon shrinkage values $\leq 20 \%$ were considered acceptable. ${ }^{20}$

Correlations between covariates and pharmacokinetic parameters were considered in the covariate model to explain interindividual variability in the golimumab pharmacokinetics. The impact of ATG on golimumab clearance was evaluated using a time-invariant approach (dichotomous 0 or 1 indicating absence or presence of ATG at the patient level $\left.\left[\mathrm{ATG}_{\text {patient }}\right]\right)$ and timevarying approaches (dichotomous at the sample level $\left[\mathrm{ATG}_{\text {sample }}\right]$, persistent upon initial detection $\left[\mathrm{ATG}_{\text {onset }}\right]$, and transient upon later confirmed disappearance $\left[\mathrm{ATG}_{\text {transient }}\right]$ ). The patients' fat-free mass was predicted using the semimechanistic model developed by Janmahasatian et al. ${ }^{21}$ Baseline serum albumin, C-reactive protein, and alkaline phosphatase concentrations were evaluated. Also, the Mayo endoscopic subscore at baseline, previous biological therapy, and concurrent treatment with corticosteroids, mesalazine, and azathioprine were evaluated.

The stepwise covariate modeling approach consisted of a forward addition procedure (decrease in $\mathrm{OFV} \geq 6.64, P \leq 0.010$; chi-square distribution, 1 degree of freedom) and a backward elimination procedure (increase in $\mathrm{OFV} \geq 10.8, P$ value $\leq 0.001$; chi-square distribution, 1 degree of freedom).

\section{Exposure-response model}

Pharmacokinetic and exposure-response modeling were performed sequentially. The individual predicted golimumab serum concentrations and cumulative areas under the golimumab concentration-time curve at days $14,28,42$, and 98 were obtained from the empirical Bayesian estimates and individual dosing histories during estimation of the parameters of the final pharmacokinetic model. A Markov model then was implemented to explore the relationship between these golimumab exposure metrics and the probability of achieving endoscopic remission at day 98 (Fig. 1). The golimumab exposure metrics were modeled to affect the transition probabilities between Mayo endoscopic subscores of 3, 2, and combined 1 and 0 (representing endoscopic remission). A detailed description of the exposure-response model is given by Dreesen et al. ${ }^{22}$

\section{Model evaluation}

The goodness of fit of the pharmacokinetic model was assessed using plots of the observed vs population- and individual-predicted golimumab serum concentrations and plots of conditional weighted residuals vs population-predicted golimumab serum concentrations and time. Final pharmacokinetic model evaluation was performed using a predictioncorrected visual predictive check (pcVPC) by 1000 simulated data sets. Shark plots were generated to investigate how the OFV differences between the base and the final pharmacokinetic and exposure-response models are distributed across individuals. Standard errors for the parameter estimates were obtained from the covariance step. As the bootstrap is limited in use due to the unbalanced sampling design of the study, sampling importance resampling (SIR) was adopted for parameter uncertainty estimation. ${ }^{23}$ Pharmacokinetic stochastic simulations were performed $(\mathrm{n}=1000)$ using Berkeley Madonna (version 8.318; Berkeley Madonna Inc., University of California, Berkeley, CA, USA).

\section{Statistical Analyses}

Data from the 3 studies were imported in $\mathrm{R}$ (version 3.5.1; R Foundation for Statistical Computing, R Core Team, Vienna, Austria) for data wrangling, visualization, and statistical analyses. ${ }^{24}$ Graphics were generated using the ggplot2

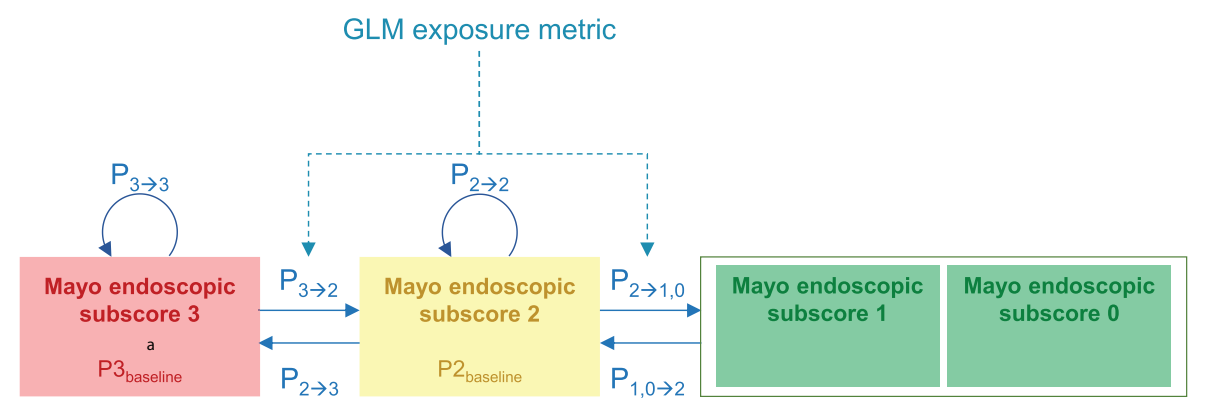

FIGURE 1. Schematic representation of the golimumab exposure-response Markov model. The 3 observed states and 6 transitions were described using 4 parameters: the baseline proportions of patients with Mayo endoscopic subscores of 3 and 2 (ie, $P 3_{\text {baseline }}$ and $P 2_{\text {baseline }}$ ) and the transition probabilities corresponding to going from a baseline Mayo endoscopic subscore of 3 to 2 (ie, $P_{3} \rightarrow_{2}$ ) and from 2 to 1 or 0 (ie $P_{2} \rightarrow{ }_{1,0}$ ). All other transition probabilities are implicit: $\mathrm{P}_{3} \rightarrow_{3}=1-\mathrm{P}_{2} \rightarrow_{3^{\prime}}, \mathrm{P}_{2} \rightarrow_{3}=1-\mathrm{P}_{3} \rightarrow_{2^{\prime}} \mathrm{P}_{2} \rightarrow_{2}=1-\mathrm{P}_{2} \rightarrow_{1,0}-\left(1-\mathrm{P}_{3} \rightarrow_{2}\right)$, and $\mathrm{P}_{1,0} \rightarrow_{2}=1-\mathrm{P}_{2} \rightarrow_{1,0}$. 
package in RStudio (version 1.1.383; R Studio, Inc., RStudio Team, Boston, MA, USA). ${ }^{25}$

Descriptive statistics were stated as percentages for discrete variables and as median (interquartile range) for continuous variables. ATG and biomarker concentrations below the limit of quantification were replaced with the limit of quantification. Golimumab serum concentrations below the limit of quantification were replaced with the limit of quantification for statistical analyses other than population modeling. Available case analysis was implemented to deal with missing endoscopy data. The Fisher exact test was used for the analysis of discrete variables. The Wilcoxon signed rank test was used for analysis of paired measurements. Unpaired data were analyzed with the Wilcoxon rank sum test. Diagnostic performance was assessed with receiver operating characteristic curve (ROC) analysis. A therapeutic threshold value was selected using the Youden $J$ statistic. ${ }^{26}$ Generalized linear model selection was performed by heuristic searches using the glmulti $\mathrm{R}$ function based on the lowest small-sample corrected Akaike Information Criterion. ${ }^{27}$ A 2-sided $P$ value $\leq 0.050$ denoted statistical significance.

\section{ETHICAL CONSIDERATIONS}

The studies were approved by the local Ethics Committee of the University Hospitals Leuven (study 1: B322201630705/ S59372; and study 2: B322201213950/S53684) and the Republic of Slovenia National Medical Ethics Committee (study 3: 0120-013/2016-2). The prospective study (study 1) is registered at ClinicalTrials.gov with identifier NCT02910375. All studies were conducted in accordance with the principles of good clinical practice and the Declaration of Helsinki. All patients provided written informed consent before study participation.

\section{RESULTS}

\section{Data Source}

A total of 56 adult patients with UC were included for the population pharmacokinetic model building (Table 1). Golimumab serum concentrations were obtained from 710 blood samples (296 DBS samples and 414 peripheral venipuncture samples) (Supplementary Fig. 1). None of the samples had a golimumab serum concentration below the limit of quantification. Still, ATG were detected in 15/710 samples (2\%) from $7 / 56$ patients $(13 \%)$ using the drug-tolerant assay. Eleven patients $(20 \%)$ had previously been treated with another biological (infliximab or adalimumab).

\section{Population Pharmacokinetic Analysis}

\section{Base model}

The data were best described by a 2-compartment model with linear absorption and elimination (Table 2). Including interindividual variability on the apparent linear clearance $(\mathrm{CL} / \mathrm{F})$, on the apparent volume of distribution in the central compartment $(\mathrm{V} / \mathrm{F})$, and on the apparent volume of distribution in the peripheral compartment $\left(\mathrm{V}_{\mathrm{p}} / \mathrm{F}\right)$ significantly improved the model fit. The $\eta$-shrinkage of the empirical Bayesian estimates of the interindividual random effects was $3 \%, 13 \%$, and $18 \%$ for $\mathrm{CL} / \mathrm{F}$, $\mathrm{V}_{\mathrm{c}} / \mathrm{F}$, and $\mathrm{V}_{\mathrm{p}} / \mathrm{F}$, respectively. The residual error was best described using combined additive and proportional error models for venipuncture and DBS samples separately. The $\varepsilon$-shrinkage was $7 \%$.

\section{Covariate analysis and final model}

From the univariate analysis, CL/F was shown to be significantly higher when ATG were present, when albumin was lower, and when the patient was previously treated with another biological $(P<0.0001, P=0.019$, and $P=0.028$, respectively $)$ (Supplementary Table 1). $\mathrm{V}_{\mathrm{p}} / \mathrm{F}$ was significantly higher in patients who had previously been treated with another biological $(P<0.0001)$. After stepwise forward addition and backward elimination, ATG and previous biological therapy were found to correlate significantly with $\mathrm{CL} / \mathrm{F}$ and $\mathrm{V}_{\mathrm{p}} / \mathrm{F}$, respectively, and thus remained in the final model. The apparent golimumab clearance and volume of distribution in the peripheral compartment were estimated in the final model from the following equations:

$$
\begin{array}{r}
C L / F=(0.417 \times A T G 0)+(0.545 \times A T G 1) \times e^{0.278} \\
V_{p} /_{F}=(1) \\
\end{array}
$$

where either dummy variable ATG0 (ATG absent) or ATG1 (ATG present) took the value 1 and, similarly, PREV0 (no previous biological therapy) or PREV1 (previous biological therapy) took the value 1 , whereas the other dummy variable remained at the initialized value of 0 . Golimumab clearance was $31 \%$ higher when ATG had been detected. Patients who had received previous biological therapy had a 4.0-fold higher volume of distribution in the peripheral compartment. Previous biological therapy and detection of ATG were not correlated (phi coefficient of correlation, $0.08 ; P=0.614$ ). Body weight was not identified as a significant covariate in our analysis.

\section{Evaluation of the final model}

The goodness-of-fit plots showed that the model described the observed data adequately (Supplementary Fig. 2). The pcVPC showed a good predictive capacity of the model (Fig. 2). The median and 95\% confidence intervals (CIs) obtained from SIR are reported in Table 2. Twenty-nine (52\%) patients had a decrease in the OFV with the final model vs the base model (Supplementary Fig. 3A). The simulated effects of ATG and previous biological therapy on the golimumab pharmacokinetics are shown in Figure 3. The unexplained interindividual variability remained high after the introduction of the 2 covariates $\left(57 \%\right.$ and $221 \%$ for $\mathrm{CL} / \mathrm{F}$ and $\mathrm{V}_{\mathrm{p}} / \mathrm{F}$, respectively). 
TABLE 2. Golimumab Population Pharmacokinetic and Pharmacodynamic Parameter Estimates

\begin{tabular}{|c|c|c|c|c|}
\hline \multirow[b]{2}{*}{ Parameter } & \multirow{2}{*}{$\begin{array}{l}\frac{\text { Base Model }}{\text { Estimate }(\% \text { RSE) }} \\
{[\% \text { Shrinkage }]}\end{array}$} & \multicolumn{3}{|c|}{ Final Model } \\
\hline & & $\begin{array}{l}\text { Estimate }(\% \mathrm{RSE}) \\
{[\% \text { Shrinkage }]}\end{array}$ & SIR Estimate (SIR 95\% CI) & $\begin{array}{c}\text { SIR } \\
\text { Deviation, } \%\end{array}$ \\
\hline Pharmacokinetic model & OFV 957.9 & OFV 922.6 & & \\
\hline \multicolumn{5}{|l|}{ Typical values } \\
\hline $\mathrm{k}_{\mathrm{a}}, \mathrm{d}^{-1}$ & $0.473(15)$ & $0.495(15)$ & $0.501(0.417$ to 0.596$)$ & +1.21 \\
\hline $\mathrm{CL} / \mathrm{F}, \mathrm{L} / \mathrm{d}$ & $0.442(8)$ & - & $\begin{array}{l}\text { to } \\
\text { to }\end{array}$ & - \\
\hline When no ATG & - & $0.417(9)$ & $0.413(0.370$ to 0.464$)$ & -0.96 \\
\hline When ATG & - & $0.545(11)$ & $0.541(0.464$ to 0.627$)$ & -0.73 \\
\hline $\mathrm{V}_{\mathrm{c}} / \mathrm{F}, \mathrm{L}$ & $8.64(8)$ & $8.82(8)$ & $8.88(7.79$ to 9.83$)$ & +0.68 \\
\hline $\mathrm{Q} / \mathrm{F}, \mathrm{L} / \mathrm{d}$ & $0.430(25)$ & $0.469(20)$ & $0.461(0.368$ to 0.587$)$ & -1.71 \\
\hline $\mathrm{V}_{\mathrm{p}} / \mathrm{F}, \mathrm{L}$ & $4.48(35)$ & - & to & - \\
\hline When no PREV & - & $3.85(43)$ & $3.93(3.03$ to 5.18$)$ & +2.08 \\
\hline When PREV & - & $15.3(43)$ & $15.2(8.48$ to 31.0$)$ & -0.65 \\
\hline $\begin{array}{l}\text { Dried blood spot-to- } \\
\text { venipuncture conversion factor }\end{array}$ & $4.09(3)$ & $4.14(3)$ & $4.14(3.95$ to 4.34$)$ & 0.00 \\
\hline \multicolumn{5}{|l|}{ Interindividual variability } \\
\hline $\mathrm{CL}, \%$ & $58.7(12)[3]$ & $56.6(13)[4]$ & 57.1 (45.0 to 71.8$)$ & +0.88 \\
\hline $\mathrm{V}_{\mathrm{c}} / \mathrm{F}, \%$ & $29.4(15)[13]$ & $28.3(16)[14]$ & $28.6(22.4$ to 36.1$)$ & +1.06 \\
\hline $\mathrm{V}_{\mathrm{p}} / \mathrm{F}, \%$ & $169.0(17)[18]$ & $220.7(22)[21]$ & $240.4(133.7$ to 419.8$)$ & +8.93 \\
\hline $\operatorname{Corr}\left(\mathrm{CL} / \mathrm{F}-\mathrm{V}_{\mathrm{c}} / \mathrm{F}\right), \%$ & $38.1(39)$ & $37.9(43)$ & $37.2(13.7$ to 64.1$)$ & -1.85 \\
\hline $\operatorname{Corr}\left(\mathrm{CL} / \mathrm{F}-\mathrm{V}_{\mathrm{p}} / \mathrm{F}\right), \%$ & $-39.9(45)$ & $-43.0(31)$ & $-43.5(-64.1$ to -16.8$)$ & +1.16 \\
\hline $\operatorname{Corr}\left(\mathrm{V}_{\mathrm{c}} / \mathrm{F}-\mathrm{V}_{\mathrm{p}} / \mathrm{F}\right), \%$ & $58.5(24)$ & $48.6(32)$ & 48.3 (21.6 to 72.5$)$ & -0.62 \\
\hline \multicolumn{5}{|l|}{ Residual errors } \\
\hline \multicolumn{5}{|l|}{ Venipuncture } \\
\hline Proportional error, \% & $2.43(19)[7]$ & $2.62(20)[7]$ & $2.61(1.74$ to 3.66$)$ & -0.38 \\
\hline Additive error, mg/L & $0.976(28)[7]$ & $0.874(34)[7]$ & $0.886(0.650$ to 1.117$)$ & +1.37 \\
\hline \multicolumn{5}{|l|}{ Dried blood spot } \\
\hline Proportional error, \% & $5.38(19)[7]$ & $6.00(6)[7]$ & $6.04(4.79$ to 7.17$)$ & +0.67 \\
\hline Additive error, mg/L & $0.134(97)[7]$ & 0.059 (104) [7] & $0.060(0.025$ to 0.136$)$ & +1.69 \\
\hline Exposure-response model & OFV 143.0 & OFV 121.5 & & \\
\hline $\mathrm{C}_{98,50}{ }^{3 \rightarrow 2}, \mathrm{mg} / \mathrm{L}$ & - & $0.5(48)$ & $0.5(0.2$ to 1.0$)$ & 0.00 \\
\hline $\mathrm{C}_{98,50}{ }^{2 \rightarrow 1,0}, \mathrm{mg} / \mathrm{L}$ & - & $4.1(36)$ & $4.2(2.0$ to 8.1$)$ & +2.44 \\
\hline
\end{tabular}

Hill's coefficients were fixed to 1 in the exposure-response model.

Abbreviations: -, not applicable; $\mathrm{C}_{98,50}{ }^{3 \rightarrow 2}$, predicted golimumab serum trough concentration at day $98\left(\mathrm{C}_{98}\right)$ corresponding to a $50 \%$ probability of going from a Mayo endoscopic subscore of 3 to $2 ; \mathrm{C}_{98,50}{ }^{2 \rightarrow 1,0}$, predicted golimumab serum trough concentration at day $98\left(\mathrm{C}_{98}\right)$ corresponding to a $50 \%$ probability of going from a Mayo endoscopic subscore of 2 to 1 or 0; Corr, correlation; $\mathrm{k}_{\mathrm{a}}$, absorption rate constant; PREV, previous biological therapy; $\mathrm{Q} / \mathrm{F}$, apparent intercompartmental clearance; RSE, relative standard error.

\section{Exposure-Response Analysis}

A total of $40 / 56$ patients $(71 \%)$ underwent endoscopies both at baseline and at week 14 with evaluation of the Mayo endoscopic subscore. Endoscopic remission was achieved in $14 / 40$ patients (35\%) (Supplementary Fig. 4). These patients had higher observed and model-predicted golimumab serum trough concentrations from week 4 onwards (Supplementary Table 2). A golimumab serum trough concentration threshold at week 6 of $7.4 \mathrm{mg} / \mathrm{L}$ was identified to predict endoscopic remission at week 14 , with a positive predictive value of $83 \%$ and a negative predictive value of $82 \%$ (Fig. 4). In addition, the estimated area under the golimumab concentration-time curve from week 0 to week 6 was higher when endoscopic remission was achieved $(P=0.015)$ (Supplementary Table 2).

The predicted golimumab serum trough concentration at week 14 was identified as the best predictor of endoscopic remission (lowest OFV) (Table 2). Thirty-two (80\%) patients had a decrease in the OFV with the final model vs the base model (Supplementary Fig. 3B). The different exposure metrics were 


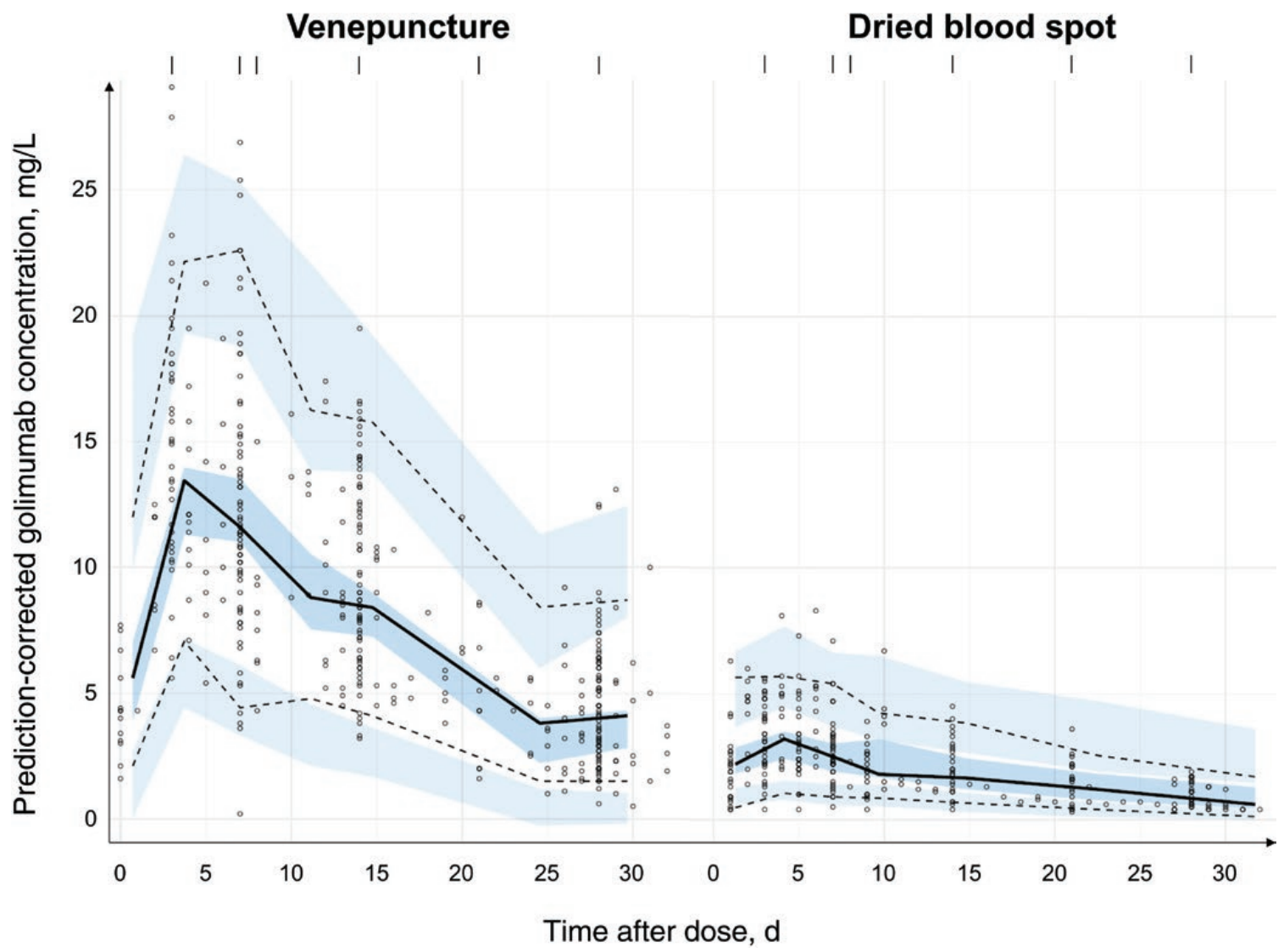

FIGURE 2. Prediction-corrected visual predictive check. The solid line connects the observed median prediction-corrected golimumab concentrations per bin. The dashed lines connect the fifth and 95th percentiles of the prediction-corrected observations. The light and dark shaded areas indicate the $90 \%$ prediction intervals of the fifth and 95 th percentiles and the median, respectively, of the simulated data $(n=1000)$. The observed prediction-corrected golimumab concentrations are represented by open circles.

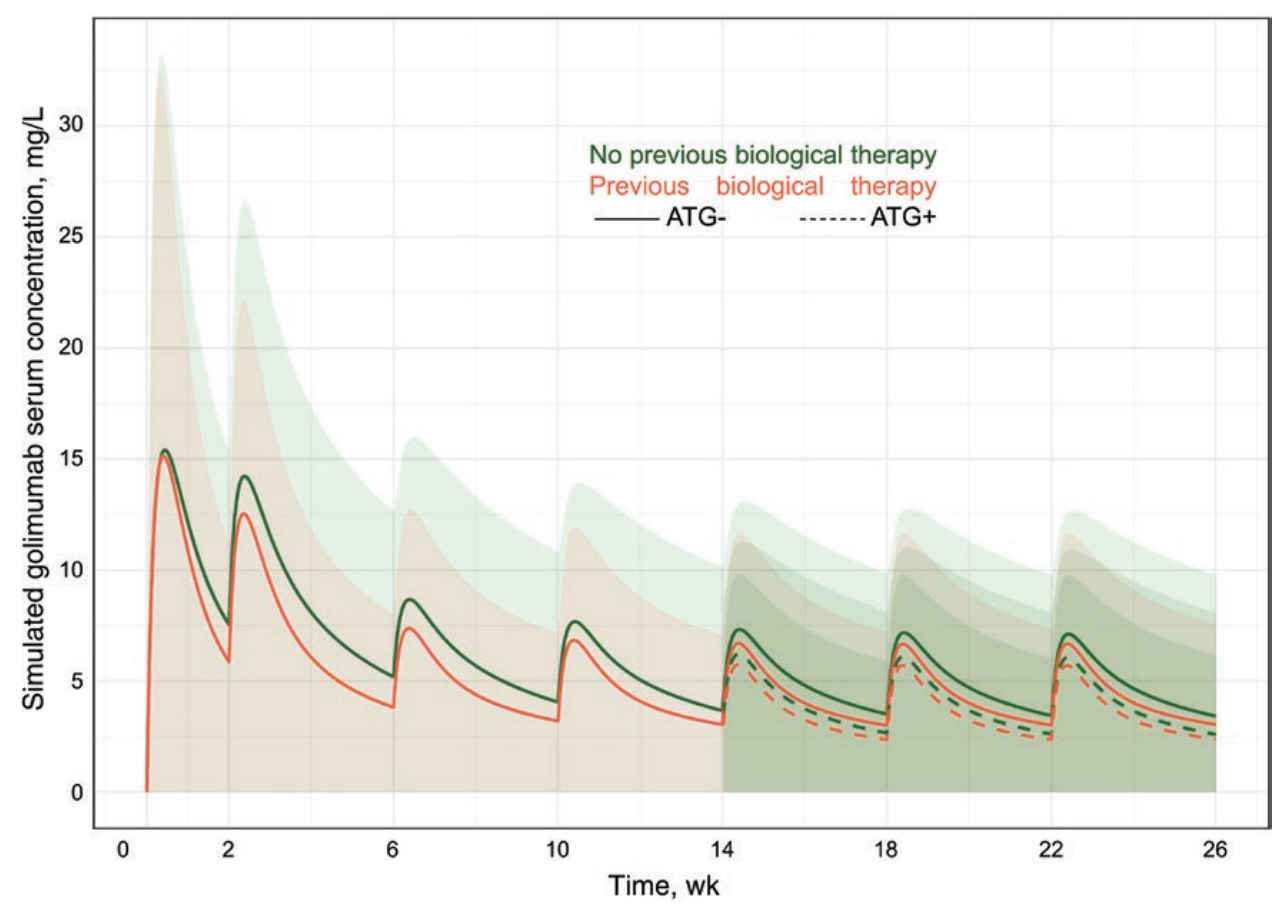

FIGURE 3. Simulated golimumab serum concentration-time profiles demonstrating the impact of previous biological therapy and antibodies to golimumab on golimumab exposure after standard induction and 100-mg maintenance dosage regimen. Data represent the median and $95 \%$ pre- 7 diction interval of 1000 simulated patients. 


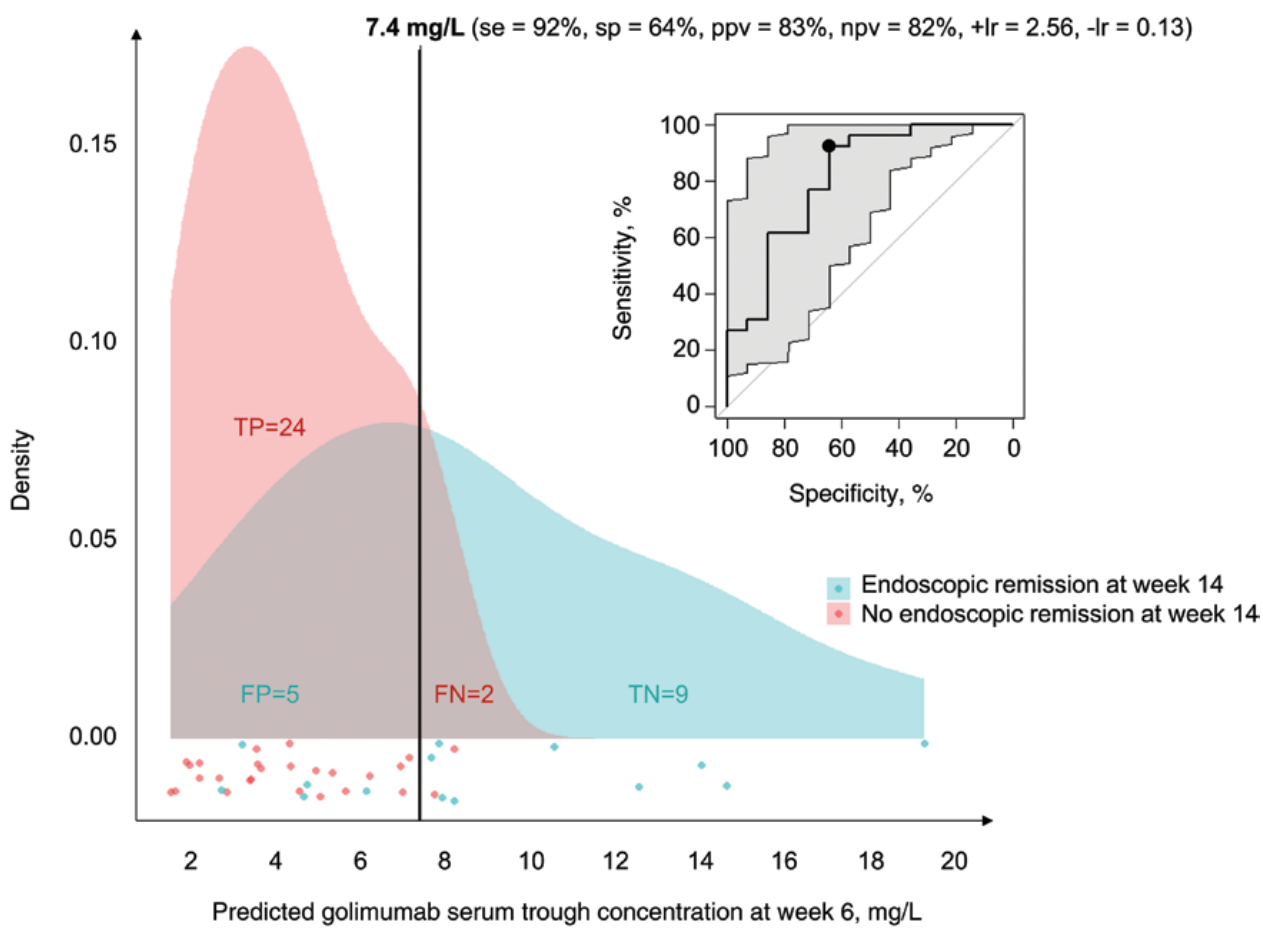

FIGURE 4. The kernel density plot represents the distributions of predicted golimumab serum trough concentrations at week 6 in patients who achieved endoscopic remission at week 14 (green) and those who did not (red). The vertical line indicates the threshold of $7.4 \mathrm{mg} / \mathrm{L}$, established using Youden's $J$ statistic. The numbers of true positive (TP), false positive (FP), true negative (TN), and false negative (FN) patients for this threshold are indicated, as are the sensitivity (se), specificity (sp), positive predictive value (ppv), negative predictive value (npv), positive likelihood ratio (+Ir), and negative likelihood ratio (-Ir). The receiver operating characteristics curve. The gray shaded area represents the $95 \%$ confidence interval of the specificity based on 2000 bootstrap replicates.

highly correlated (Spearman's $\rho>0.5$ ) (Supplementary Fig. 5). Following the label-recommended induction dosing scheme, a week 14 golimumab serum trough concentration of $3.2 \mathrm{mg} / \mathrm{L}$ was estimated to yield $41 \%$ of patients attaining endoscopic remission (Fig. 5). Similarly, targeting a week 6 golimumab serum trough concentration of $7.4 \mathrm{mg} / \mathrm{L}$ predicted $44 \%$ of patients attaining endoscopic remission (data not shown). Only $11 / 40(28 \%)$ and $18 / 40(45 \%)$ patients in our cohort reached these week 6 and 14 serum trough concentration targets, with negative predictive values of $82 \%$ and $67 \%$, respectively (Supplementary Table 2).

\section{Predictors of Response}

A Mayo endoscopic subscore of 3, a lower serum albumin at baseline, and concurrent treatment with corticosteroids were identified as the most important predictors compromising an individual's probability of achieving endoscopic remission (Supplementary Table 3, Supplementary Fig. 6).

\section{DISCUSSION}

This is the first real-world population pharmacokineticpharmacodynamic study of golimumab in adult patients with UC. The pooled golimumab serum concentration-time data of 56 patients were adequately described by a 2-compartment model with first-order absorption and elimination. The availability of a validated DBS micro-sampling method facilitated frequent sampling while limiting the patient burden, thereby aiding the identification of the peripheral compartment, which was not identified by $\mathrm{Xu}$ et al., who relied on the relatively sparse sample collection from the PURSUIT and GO-KIDS programs. ${ }^{10}$ As DBS can be self-collected by the patient, requires no centrifugation, and can be transported at room temperature, it is a convenient field work substitute for serum sampling in clinical studies. ${ }^{11}$

The estimated values of the golimumab pharmacokinetic parameters are generally consistent with those reported in literature. ${ }^{10,15,18,19}$ The DBS-to-venipuncture conversion factor estimated from the model was $4.1 \% \pm 3 \%$ and was similar to that obtained from the regression analysis by Detrez et al. ${ }^{11}$ ATG and previous biological therapy were found to increase the golimumab apparent clearance and the volume of distribution in the peripheral compartment, respectively. Despite the statistical significance of the covariate effects on the golimumab pharmacokinetics, the remaining unexplained interindividual variability remains large, opposing a priori dose stratification.

In the study by Xu et al., the presence of ATG increased golimumab clearance by $21 \%$, which is less than the $31 \%$ in 


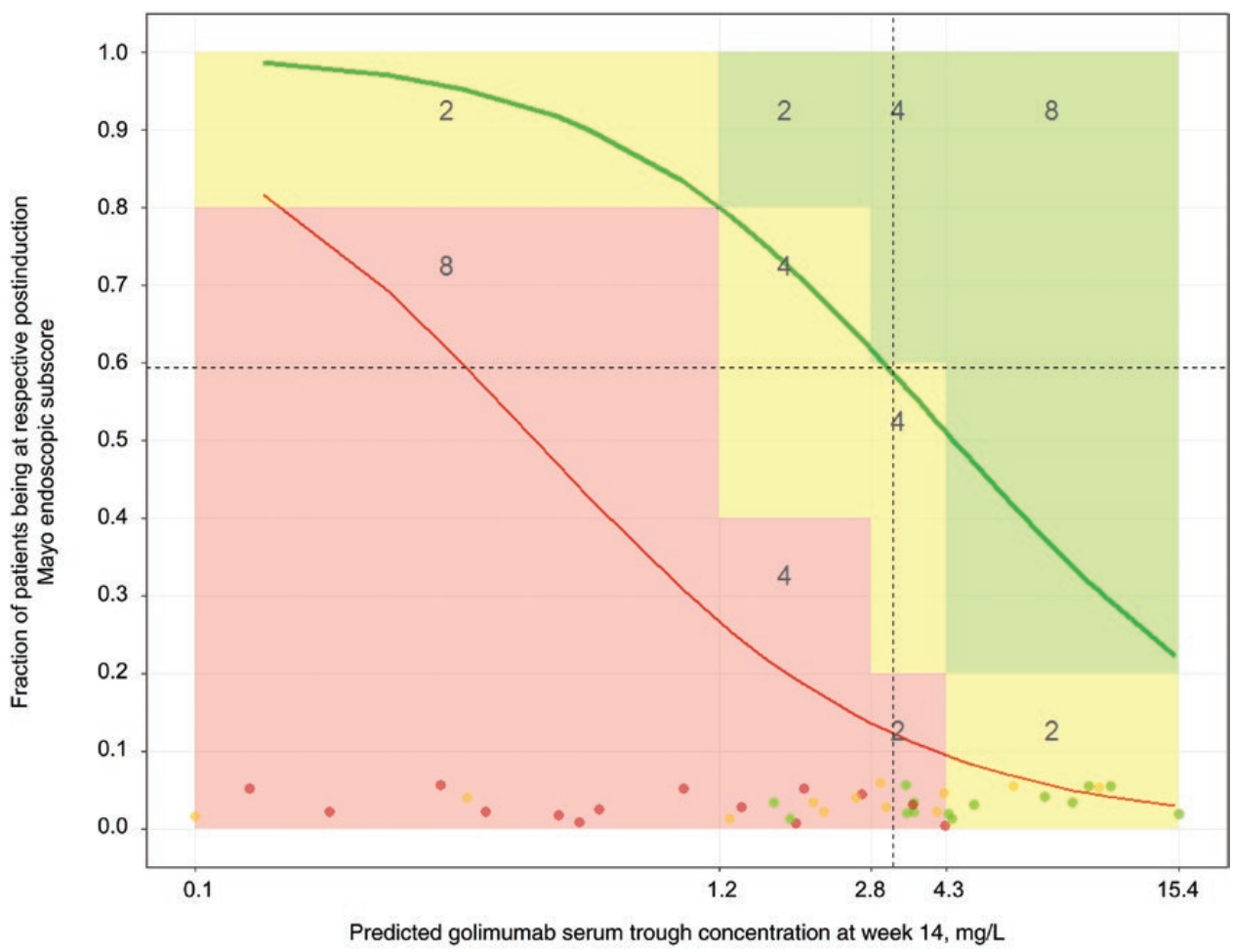

FIGURE 5. Goodness of fit of the exposure-response model. Observed (tiles) fractions of patients attaining postinduction Mayo endoscopic subscores of 3 (red), 2 (yellow), and $\leq 1$ (green) and predicted (lines) fractions of patients attaining postinduction Mayo endoscopic subscores of 3 (red) and $>1$ (green) as a function of the predicted golimumab serum trough concentration at week 14 ( 4 quantiles). Gray numbers indicate the number of patients in each bin.

our study. ${ }^{10}$ However, they used a drug-sensitive ATG assay, whereas we applied a drug-tolerant ATG assay. ${ }^{6}{ }^{19}$ Nonetheless, the effect of ATG on golimumab clearance cannot be compared because different calibrators were used, different strategies were used for sample selection, and other modeling approaches were used (see the "Methods" section). ${ }^{28,29}$ Although Xu et al. classified patients as ATG positive or negative depending on the detection of ATG at any visit (time-constant approach), we considered a time-varying approach, where patients were ATG positive once ATG were detected, until later proof of the absence of ATG. Taking into account the transiency in ATG detection resulted in the largest drop in OFV in the univariate analysis.

Although often suggested, we have now confirmed that previous biological therapy negatively affects the golimumab pharmacokinetics, and thereby also the response. ${ }^{9}$ Our findings demonstrate the importance of inclusion of these patients in future clinical drug development programs. Although the 200/100-mg and 400/200-mg induction schemes resulted in similar proportions of biologically naïve PURSUIT-SC patients attaining the efficacy end points, patients previously treated with another biological may benefit from higher induction dosing. ${ }^{3}$ There is currently no biological reason for the larger volume of distribution in the peripheral compartment in patients previously treated with another biological, but this may be explained by a larger in-tissue TNF $\alpha$ load or protein-losing enteropathy. ${ }^{30,31}$ None of the available variables in our cohort correlated with previous biological therapy (all $P>0.050$, data not shown). Further studies are needed to investigate the underlying mechanism in detail.

Unlike the model of $\mathrm{Xu}$ et al., body weight was not an influential covariate of the golimumab pharmacokinetics in our analysis. ${ }^{10}$ This may be explained by the relatively limited variability in body weights in our cohort, as compared with the cohort of Xu et al., which also included pediatric data. In addition, we did not identify the effects of comedication and serum albumin.

Sixty-five percent of the patients in our cohort did not achieve endoscopic remission at week 14 , as compared with the $58 \%$ of patients not achieving endoscopic remission at week 6 in PURSUIT-SC. ${ }^{3}$ Patients with endoscopic remission were found to have higher golimumab exposure. By performing ROC analysis, as was also done by Adedokun et al., we identified a golimumab serum trough concentration threshold of $7.4 \mathrm{mg} / \mathrm{L}$ at week 6 , which is much higher than the previously suggested $2.5 \mathrm{mg} / \mathrm{L}$ for targeting clinical response. ${ }^{5}$ Only $28 \%$ of the patients in our study attained the $7.4-\mathrm{mg} / \mathrm{L}$ target, suggesting the potential benefits of intensified golimumab induction dosing. In addition, the poor prognosis when below this 
threshold (17\% endoscopic remission) and the good prognosis when above this threshold ( $82 \%$ endoscopic remission) support a role for TDM for targeting this threshold. Similarly, we identified a golimumab serum concentration threshold at week 4 of $11.4 \mathrm{mg} / \mathrm{L}$, which is again higher than the $7.4-\mathrm{mg} / \mathrm{L}$ threshold for clinical response proposed by Adedokun et al. ${ }^{5}$

Our exposure-response model was informed by individual predicted golimumab serum concentrations or cumulative areas under the golimumab concentration-time curves at days 28, 42, and 98 after the start of induction therapy. These exposure metrics informed the probabilities of transitioning from (i) a baseline Mayo endoscopic subscore of 3 to a subscore of 2 at week 14 and (ii) a baseline Mayo endoscopic subscore of 2 to a subscore $\leq 1$ at week 14 . The OFV dropped with 21.5 points from the null model, where transition probabilities were estimated by chance alone, to the model informed by the individual predicted golimumab serum trough concentration at week 14, which supports a role for exposure optimization by means of TDM.

Our population pharmacokinetic and exposure-response models allow dose selection for targeting a certain golimumab exposure and the associated probability of endoscopic remission. However, as the interindividual variability remains largely unexplained, Bayesian methodology can be used for better estimating the individual patients' model parameters. Therefore, early sampling is required, which may be facilitated by DBS or another validated micro-sampling technique (eg, volumetric absorptive micro-sampling), and implementation of the model in a dedicated TDM software tool is a requisite.

As opposed to the currently used "one dose fits all" approach, our suggested "one exposure fits all" approach gives all patients a more equal chance of achieving endoscopic remission. Model-based golimumab dose optimization thus provides a more justifiable approach for facilitating the decision on drug continuation. However, in patient populations with higher proportions of patients previously treated with biologicals, higher doses will be needed, as these patients need more drug to achieve the same exposure. In addition, in a population consisting of more patients with a baseline Mayo endoscopic subscore of 3 , higher doses are needed, as more patients need additional exposure for transitioning from subscore of 3 to 2 .

The results of our analyses must be interpreted carefully. First, the sample size of the study was relatively small as compared with the PURSUIT studies. Although rich sampling resulted in high precision of parameter estimates, 56 patients seemed to be insufficient for detecting previously identified effects of serum albumin and body weight. Second, our model assumes time-invariant pharmacokinetics (apart from the ATG effect), although it has been shown that the infliximab pharmacokinetics can change with disease activity. ${ }^{32}$ Future studies should consider evaluating time-varying serum albumin for capturing the negative effect of the inflammatory burden on golimumab exposure. Third, our population pharmacokinetic model was built using data from patients who all received the standard golimumab induction therapy. Therefore, our model does not support interval shortening and dose escalations, resulting in a golimumab exposure outside the range observed in this study. Fourth, the golimumab assay used by $\mathrm{Xu}$ et al. detected significantly lower concentrations as compared with the assay used in our study. ${ }^{29}$ Despite an excellent correlation between both assays, one cannot directly compare measurements and should thus be careful when comparing therapeutic thresholds between the studies. Also, the ATG assay comparison was inconclusive. ${ }^{29}$ Furthermore, no ATG were detected within the first 14 weeks of therapy, but this might be biased by our sample selection strategy (see the "Methods"). Finally, the currently available dose strengths of $50 \mathrm{mg}$ and $100 \mathrm{mg}$ limited the flexibility of golimumab dose individualization. Doseadjustable delivery systems may be cost-effective in the future, but most urgent is the need for repeating the PURSUIT-SC study including patients in whom previous biological therapy has failed, similar to what was done in the vedolizumab GEMINI-3 and ustekinumab UNITI-2 studies. ${ }^{33,} 34$

\section{CONCLUSIONS}

To conclude, the results of our study support a role for model-based TDM during golimumab induction therapy. Higher golimumab exposure needs to be targeted for achieving endoscopic remission as compared with clinical outcomes. With the currently approved induction dosing, only $25 \%$ and $43 \%$ of patients reached the proposed $7.4-\mathrm{mg} / \mathrm{L}$ and $3.2-\mathrm{mg} / \mathrm{L}$ serum trough concentration targets at days 42 and 98 , respectively, suggesting underexposure in a substantial proportion of patients. Prospective trials are urged, as poor response to standard golimumab induction therapy compromises its future use in clinical practice.

\section{ACKNOWLEDGMENTS}

We thank Carmen Sekulic Bobnar and Tadeja Polanc for the recruitment of patients and Sophie Tops, Griet Compernolle, and Els Brouwers for an excellent job in maintaining the Biobank database and measuring the drug and antidrug antibody concentrations.

\section{REFERENCES}

1. Shealy DJ, Cai A, Staquet K, et al. Characterization of golimumab, a human monoclonal antibody specific for human tumor necrosis factor $\alpha$. MAbs. 2010;2:428-439.

2. Rutgeerts P, Feagan BG, Marano CW, et al; PURSUIT-IV Study Group Randomised clinical trial: a placebo-controlled study of intravenous golimumab induction therapy for ulcerative colitis. Aliment Pharmacol Ther. 2015;42:504-514.

3. Sandborn WJ, Feagan BG, Marano C, et al; PURSUIT-SC Study Group. Subcutaneous golimumab induces clinical response and remission in patients with moderate-to-severe ulcerative colitis. Gastroenterology. 2014;146:85-95; quiz e14.

4. Sandborn WJ, Feagan BG, Marano C, et al; PURSUIT-Maintenance Study Group. Subcutaneous golimumab maintains clinical response in patients with moderate-to-severe ulcerative colitis. Gastroenterology. 2014;146:96-109.e1

5. Adedokun OJ, Xu Z, Marano CW, et al. Pharmacokinetics and exposure-response relationship of golimumab in patients with moderately-to-severely active ulcerative colitis: results from phase $2 / 3$ PURSUIT induction and maintenance studies. J Crohns Colitis. 2017;11:35-46. 
6. Detrez I, Dreesen E, Van Stappen T, et al. Variability in golimumab exposure: a 'real-life' observational study in active ulcerative colitis. J Crohns Colitis. 2016;10:575-581.

7. Peyrin-Biroulet L, Sandborn W, Sands BE, et al. Selecting therapeutic targets in inflammatory bowel disease (STRIDE): determining therapeutic goals for treatto-target. Am J Gastroenterol. 2015;110:1324-1338.

8. Colombel JF, Rutgeerts P, Reinisch W, et al. Early mucosal healing with infliximab is associated with improved long-term clinical outcomes in ulcerative colitis. Gastroenterology. 2011;141:1194-1201.

9. Singh S, George J, Boland BS, et al. Primary non-response to tumor necrosis factor antagonists is associated with inferior response to second-line biologics in patients with inflammatory bowel diseases: a systematic review and meta-analysis. J Crohns Colitis. 2018;12:635-643.

10. Xu Y, Adedokun OJ, Chan D, et al. Population pharmacokinetics and exposureresponse modeling analyses of golimumab in children with moderately to severely active ulcerative colitis. J Clin Pharmacol. 2019;59:590-604.

11. Detrez I, Schops G, Lefrère J, et al. Golimumab dried blood spot analysis (GOUDA): a prospective trial showing excellent correlation with venepuncture samples and more detailed pharmacokinetic information. AAPS J. 2018;21:1-9.

12. Stefanovic S, Detrez I, Compernolle G, et al. P632 Trough levels of golimumab at week 6 predict drug retention rate in ulcerative colitis. J Crohn's Colitis. 2018;12:S429-S430.

13. Gislefoss RE, Grimsrud TK, Mørkrid L. Stability of selected serum proteins after long-term storage in the Janus Serum Bank. Clin Chem Lab Med. 2009; 47:596-603.

14. Kothare PA, Bateman KP, Dockendorf M, et al. An integrated strategy for implementation of dried blood spots in clinical development programs. Aaps J. 2016;18:519-527.

15. Adedokun OJ, Xu Z, Liao S, et al. Sa1935 population pharmacokinetic modeling analysis of golimumab in adult patients with moderately to severely active ulcerative colitis. Gastroenterology. 2016;150:S408.

16. $\mathrm{Hu} \mathrm{C}, \mathrm{Xu} \mathrm{Z}$, Zhang $\mathrm{Y}$, et al. Population approach for exposure-response modeling of golimumab in patients with rheumatoid arthritis. J Clin Pharmacol. 2011;51:639-648.

17. $\mathrm{Xu} \mathrm{ZH}$, Lee H, Vu T, et al. Population pharmacokinetics of golimumab in patients with ankylosing spondylitis: impact of body weight and immunogenicity. Int J Clin Pharmacol Ther. 2010;48:596-607.

18. Xu Z, Vu T, Lee H, et al. Population pharmacokinetics of golimumab, an antitumor necrosis factor-alpha human monoclonal antibody, in patients with psoriatic arthritis. J Clin Pharmacol. 2009;49:1056-1070.
19. Zhou H, Jang H, Fleischmann RM, et al. Pharmacokinetics and safety of golimumab, a fully human anti-TNF-alpha monoclonal antibody, in subjects with rheumatoid arthritis. J Clin Pharmacol. 2007;47:383-396.

20. Savic RM, Karlsson MO. Importance of shrinkage in empirical Bayes estimates for diagnostics: problems and solutions. AAPS J. 2009;11:558-569.

21. Janmahasatian S, Duffull SB, Ash S, et al. Quantification of lean bodyweight. Clin Pharmacokinet. 2005;44:1051-1065.

22. Dreesen E, Faelens R, Van Assche G, et al. Optimising infliximab in duction dosing for patients with ulcerative colitis. $\mathrm{Br} \mathrm{J}$ Clin Pharmacol. 2019;85:782-795

23. Dosne AG, Bergstrand M, Karlsson MO. An automated sampling importance resampling procedure for estimating parameter uncertainty. $J$ Pharmacokine Pharmacodyn. 2017;44:509-520.

24. R Core Team. R: A Language and Environment for Statistical Computing [com puter program]. Vienna, Austria: R Foundation for Statistical Computing; 2018.

25. Wickham H. ggplot2: Elegant Graphics for Data Analysis. New York: SpringerVerlag; 2016.

26. Robin X, Turck N, Hainard A, et al. Proc: an open-source package for R and S+ to analyze and compare ROC curves. BMC Bioinformatics. 2011;12:1-8.

27. Calcagno V. Glmulti: Model Selection and Multimodel Inference Made Easy. 2012 https://cran.r-project.org/package=glmulti

28. Gils A, Van Stappen T, Dreesen E, et al. Harmonization of infliximab and antiinfliximab assays facilitates the comparison between originators and biosimilars in clinical samples. Inflamm Bowel Dis. 2016;22:969-975.

29. Paul S, Duru G, De Vries A, et al. P226 Comparison of four different immunoassays for measuring golimumab and anti-golimumab antibody concentration in patients with ulcerative colitis. J Crohn's Colitis. 2017;11:S192.

30. Yarur AJ, Jain A, Sussman DA, et al. The association of tissue anti-TNF drug levels with serological and endoscopic disease activity in inflammatory bowel disease: the ATLAS study. Gut. 2016;65:249-255.

31. Brandse JF, Van Den Brink GR, Wildenberg ME, et al. Loss of infliximab into feces is associated with lack of response to therapy in patients with severe ulcerative colitis. Gastroenterology. 2015;149:350-355.e2.

32. Kevans D, Murthy S, Mould DR, Silverberg MS. Accelerated clearance of infliximab is associated with treatment failure in patients with corticosteroidrefractory acute ulcerative colitis. J Crohns Colitis. 2018;12:662-669.

33. Sands BE, Feagan BG, Rutgeerts P, et al. Effects of vedolizumab induction therapy for patients with Crohn's disease in whom tumor necrosis factor antagonist treatment failed. Gastroenterology. 2014;147:618-627.e3.

34. Feagan BG, Sandborn WJ, Gasink C, et al; UNITI-IM-UNITI Study Group. Ustekinumab as induction and maintenance therapy for Crohn's disease. $N$ Engl J Med. 2016;375:1946-1960. 